\title{
Mecanismos de control de la plataforma Uber sobre sus conductores asociados en la Región Metropolitana de Chile
}

\author{
Control mechanisms of Uber platform over its associate drivers in the Metropolitan Region \\ of Chile
}

\section{Osvaldo Vallejos Rivero \\ Universidad de Chile, Chile}

\begin{abstract}
RESUMEN En el presente artículo se describen y analizan los principales mecanismos de control implementados por la plataforma Uber en el proceso de trabajo de sus conductores asociados en la Región Metropolitana de Chile. La información fue producida a través de 12 entrevistas realizadas a conductores de Uber residentes en la Región Metropolitana entre septiembre de 2019 y febrero de 2020, analizadas a través de un análisis cualitativo de contenido con el software Atlas ti7. El marco analítico utilizado fue tomado principalmente de la denominada teoría del proceso de trabajo, incorporando además ciertas discusiones actuales sobre nuevas formas de control elaboradas específicamente para el contexto del trabajo en plataformas digitales. Los principales resultados muestran una combinación entre elementos de control persistentes a lo largo del tiempo y elementos novedosos incorporados en los últimos años, específicamente a través de una introducción de mayor eficacia a métodos de control antiguos gracias a las nuevas tecnologías, haciendo que tanto las formas nuevas como antiguas de control sean más instantáneas, opacas y abarcadoras. Con todo, se observa una predominancia de aquellos mecanismos de control asociados al control algorítmico y a la estrategia de autonomía responsable.
\end{abstract}

PALABRAS CLAVE Control; proceso de trabajo; conductores de Uber. 
ABSTRACT This article discusses the main control mechanisms implemented by Uber platform in the labour process of its associated drivers in the Metropolitan Region of Chile. The information was gleaned from 12 interviews with Uber drivers residing in the Metropolitan Region between September 2019 and February 2020. It was analyzed through qualitative content analysis with Atlas ti7 software. The analytical framework was mainly taken from the labour process theory; current discussions about new forms of control, developed especifically in the context of digital platforms, were incorporated. The main results show a combination between persistent control elements over time and novel elements incorporated in recent years, specifically through a more efficient introduction of old control methods thanks to new technologies, making both new and old forms of control more instantaneous, opaque and comprehensive. However, there is a predominance of those control mechanisms associated to algorithmic control and the strategy of responsible autonomy.

KEY WORDS Control; labour process; Uber drivers.

\section{Introducción}

En este artículo se describen y analizan las principales formas de control implementadas por la plataforma Uber sobre el trabajo de sus conductores asociados, identificadas en el discurso de estos mismos conductores. Los resultados aquí presentados son parte de una investigación titulada "Control y resistencias en el proceso de trabajo de los conductores de Uber de la Región Metropolitana", realizada como memoria de título para obtener el grado de sociólogo de la Universidad de Chile.

La expansión de las plataformas digitales como Uber ha tenido importantes consecuencias sobre el mundo del trabajo y las relaciones laborales. Entre los cambios más importantes se cuenta el hecho de que estas plataformas eliminan los costos asociados a fuerza de trabajo, argumentando que no poseen ningún trabajador a su servicio, siendo tan solo plataformas de vinculación entre proveedores y consumidores (Todolí, 2015). Esto ha generado niveles de flexibilidad laboral inéditos, lo que se ha interpretado de forma ambivalente en torno a la tensión entre autonomía y control (Wood, 2019). Se ha argumentado a favor del trabajo en plataformas como Uber en función de esta flexibilidad y la posibilidad de acceder de forma fácil a trabajo en mercados laborales limitados (Vaclavik \& Pithan, 2018). Mientras que entre sus detractores se esgrime su naturaleza precaria producto del tipo de vínculo entre el trabajador y la empresa. Esto genera fenómenos asociados como la falta de protección laboral, bajas remuneraciones, tiempo de trabajo superior a la jornada legal, difuminación del límite entre tiempo libre y tiempo de trabajo, entre otros (De Stefano, 2016; Graham \& Hjorth, 2017). 
En Chile la principal manifestación del trabajo en plataformas se ha dado a través de las plataformas de transporte de pasajeros como Uber (Comisión Nacional de Productividad (CNP), 2018), además de plataformas de reparto de comida como Rappi o UberEats, que en conjunto poseen alrededor de 15000 trabajadores (Ríos \& Cifuentes, 2020). Dentro de las plataformas de transporte de pasajeros presentes en el país, Uber es la más importante, con alrededor de 2.3 millones de usuarios activos de la aplicación y más de 70.000 conductores presentes en todas las regiones (CNP, 2018), concentrando gran parte del mercado de transporte privado de pasajeros, razón por la cual se escoge esta empresa en particular para realizar la investigación.

En particular, uno de los elementos que más ha llamado la atención del proceso de trabajo en Uber es el tipo de control al cual son sometidos los conductores asociados a la plataforma, el que ha sido denominado como control algorítmico (Wood, 2019) o administración managerial (Möhlmann \& Zalmanson, 2017) y se caracteriza por operar de manera automatizada sobre una cantidad significativa de trabajadores remotos, rastreando y evaluando constantemente el comportamiento de estos trabajadores y aplicando sanciones o recompensas de manera automática. De la misma manera, se destaca cómo este control opera interviniendo la labor de los trabajadores en función de los objetivos de la empresa, pero sin la necesidad de controlar directamente elementos como la jornada o los lugares de trabajo. Esto va de la mano con la exaltación discursiva de elementos como la flexibilidad, la autonomía o el "ser tu propio jefe" por parte de la empresa Uber, los cuales sirven al mismo tiempo como legitimación de su modelo de organización del trabajo.

Si bien la relación establecida entre Uber y sus conductores asociados opera de manera similar en todos los países donde la plataforma se encuentra presente, posee ciertos elementos específicos para el caso chileno que son importantes de destacar. Es sabido que la legislación laboral del país es débil en cuanto a la protección y derechos de los trabajadores, lo que para los trabajadores de plataformas como Uber repercute en que hasta el momento no se ha regulado su relación laboral, ya sea incorporándolos a la normativa existente o creando figuras especiales, como ha ocurrido en países como Argentina, España o Inglaterra (Martínez \& Armijo, 2018; Williams, 2018), de manera que se encuentran desprovistos de cualquier tipo de protección al empleo y seguridad social asociada. Como se verá, esta desregulación es fundamental para la generación de ganancia de la empresa y, por lo tanto, para las formas de control que necesita implementar.

Considerando lo anterior es que surge la motivación por investigar el control ejercido por la plataforma Uber sobre sus conductores presentes en la Región Metropolitana de Chile, recogiendo los elementos señalados por otras investigaciones a lo largo del mundo acerca del tipo de control implementado por la plataforma, así como también elementos de la tradición de la teoría del proceso de trabajo, la cual ha poseído 
como una de sus preocupaciones centrales el estudio del control sobre los trabajadores. Si bien en Chile la emergencia de Uber y otras plataformas digitales ha suscitado debates con respecto a su regulación legal (CNP, 2018), las investigaciones desde las ciencias sociales son todavía escasas y no han profundizado en elementos como la organización del trabajo y la potencial conflictividad que esto suscita. Por lo que, si la sociología quiere hacerse parte de este debate, debe necesariamente introducir cuestiones asociadas a las condiciones en las que desarrollan su trabajo los trabajadores de plataformas digitales, como lo son las formas de control y las respuestas a este.

\section{El problema del control en el estudio del proceso de trabajo}

Como se señaló, el control ejercido sobre los trabajadores en el marco del desarrollo de sus labores al interior de una empresa o unidad productiva es uno de los fenómenos más estudiados en el ámbito de la sociología del trabajo, especialmente a partir de los estudios de Braverman (1983), quien inaugura la corriente denominada teoría del proceso de trabajo (labour process theory). La tesis central de esta corriente teórica señala que en el modo de producción capitalista existe un conflicto estructurado de intereses en la relación entre el capital y el trabajo. Esto debido a que, si bien queda claro para ambas partes que la fuerza de trabajo de los obreros le pertenece al capitalista durante un período específico de tiempo en cuanto mercancía intercambiada en el mercado, la cantidad exacta de trabajo que debe ser llevada a cabo por los obreros es siempre indeterminada, ya que no puede especificarse en el contrato de trabajo.

Lo anterior tiene como consecuencia que los capitalistas se enfrentan al problema de convertir la fuerza de trabajo en trabajo de la forma más rentable posible. Es en virtud de esta búsqueda de maximización de su ganancia que los capitalistas implementan diversas formas de control sobre los trabajadores, para asegurarse que el proceso de trabajo se está desarrollando de la forma más eficiente posible. En tal sentido, el control sobre el proceso de trabajo no es implementado, al menos en primera instancia, con fines disciplinarios ni como un fin en sí mismo, sino que en función de la búsqueda de maximización de la ganancia a la cual están sometidas las empresas en virtud de la constante competencia propia de la producción moderna.

Autores como Edwards (1978) y Friedman (1977) han señalado que todo sistema de control debe poseer al menos cuatro elementos, a saber: dirección sobre las tareas realizadas, evaluación de dichas tareas, sanciones o recompensas según la evaluación del trabajo e intervención sobre la organización y conflictividad de los trabajadores. Estos cuatro elementos se han combinado en diversas formas a lo largo de la historia, siendo identificadas cuatro principales manifestaciones: control simple, control técnico, control burocrático y autonomía responsable. Todas estas formas de control han sido hegemónicas en algún momento del desarrollo del capitalismo, pero coexisten y predominan según el contexto específico en el cual opera la empresa. 
En primer lugar, el control simple se caracteriza porque los capitalistas ejercen su poder de manera abierta, arbitraria y directa. El capitalista tiende a intervenir a menudo en el proceso de trabajo para asegurarse de que los trabajadores estén realizando sus labores, amenazar a aquellos que no lo están haciendo y recompensar a quienes se encuentren produciendo según el ritmo exigido. Si bien existen resabios de este tipo de control, se vuelve inviable a medida que las empresas crecen y se vuelven más complejas internamente, por lo que deben implementarse mecanismos más institucionalizados y estructurales.

Por otra parte, el control técnico se caracteriza por encontrarse incrustado y depender directamente de la tecnología utilizada por la empresa. Este tipo de control emerge cuando prácticamente la totalidad de la producción se basa en la tecnología utilizada, la cual dirige y señala el ritmo del proceso de trabajo, por lo que se entiende que el control está más allá del poder directo de la administración de la empresa. Un aspecto importante que debe tenerse en cuenta con respecto a esta forma de control, dice relación con que el tipo de tecnología seleccionada por los capitalistas no es necesariamente la más eficiente, sino que la más rentable en lo referido a la efectiva transformación de la fuerza de trabajo en trabajo real.

De la misma manera, el control burocrático se define por encontrarse incrustado en la organización social de la empresa. En específico, su característica definitoria es que las jerarquías de poder presentes en la empresa se encuentran institucionalizadas en un sistema de reglas y mandatos conocidos de antemano por todos los actores involucrados. Sin embargo, resulta claro que las tareas a realizar nunca pueden ser especificadas por completo en el contrato de trabajo, por lo que la figura del supervisor no desaparece, aunque sus funciones si se transforman, ya que bajo esta forma de control solo debe cerciorarse de que los trabajadores lleven a cabo su trabajo según las reglas establecidas por la empresa.

Finalmente, la autonomía responsable implica otorgarle cierto rango de independencia y discreción en la realización de sus labores a un grupo específico de trabajadores, manteniendo la autoridad de la dirección de la empresa a través de lograr una identificación de los trabajadores con los objetivos de esta, principalmente en lo referido al aumento de la productividad. Esto produciría que los trabajadores se comporten de manera responsable con solo un grado mínimo de supervisión, por lo que se reducen costos asociados al control de la fuerza de trabajo y se previene la emergencia de conflictividad por parte de los trabajadores.

Ahora bien, estas formas de control han sido estudiadas predominantemente para el trabajo industrial en países desarrollados, el cual está asociado a características tales como la producción industrial de mercancías, la existencia de un contrato estable a lo largo del tiempo, la subordinación a un solo empleador, con reglas claras acerca de cuáles son las tareas que cumplir y con una relativamente alta seguridad social (De la 
Garza, 2012). De manera que podría parecer no ser tan adecuado para contextos de trabajo como el de los conductores de Uber.

A pesar de lo anterior, se han desarrollado investigaciones sobre plataformas como Uber desde una perspectiva afín a la teoría del proceso de trabajo (Gandini, 2019; Kellog et al., 2020; Rosenblat \& Stark, 2016). En específico, se ha señalado que el proceso de trabajo tiene lugar dentro de la plataforma, en tanto todas las relaciones sociales que se desarrollan dentro de los límites fijados por esta se convierten en relaciones sociales de producción (Gandini, 2019). Cualquier transacción y trabajo realizado por los conductores de Uber debe hacerse según las reglas y procedimientos fijados por la plataforma y solo pueden ocurrir una vez que los conductores han ingresado a esta para comenzar a trabajar, así como también el trabajo se da por terminado cuando apagan el modo conductor y salen de ella. Al conectarse en la plataforma, los conductores se vuelven objeto de una autoridad externa que convierte las peticiones de los clientes en órdenes que deben ejecutarse, determina qué tareas deben realizarse, cuánto será el pago por el trabajo realizado y controla el desempeño del conductor. Al mismo tiempo, las formas de control identificadas por investigaciones anteriores sobre el proceso de trabajo en Uber se enmarcan también en los desarrollos de la teoría del proceso de trabajo, aun cuando no siguen al pie de la letra la tipología de control elaborada por esta.

Uno de los elementos fundamentales que se han rescatado de la teoría del proceso de trabajo para estudiar el control presente entre trabajadores de plataformas digitales refiere a la distinción entre control general y control detallado introducida por Edwards (1990) y rescatada por Wood (2019) a propósito de estas nuevas formas de empleo. Esta distinción señala que mientras el control detallado refiere principalmente a la distribución, velocidad y forma de realizar el trabajo, además de la aplicación de disciplina directa en el espacio mismo de trabajo, el control general apunta a la adaptación en términos generales de los trabajadores a los objetivos de la empresa, por lo que actúan conforme a ellos. Lo anterior es importante debido a que da cuenta de que, en ciertos tipos de trabajos, aunque no existan mecanismos directos de control sobre aspectos como los horarios o lugares, puede existir de hecho un control mayor del empleador. Este es específicamente el caso de los trabajadores de plataformas digitales asociados a la gig economy (Wood, 2019), de los cuales los conductores de Uber son parte.

En relación con lo anterior es que se han elaborado nuevas tipologías de control que pretenden actualizar las formas clásicas elaboradas por los teóricos del proceso de trabajo. De esta manera se han desarrollado los conceptos de control algorítmico y administración managerial, los cuales apuntan a los sistemas de monitoreo y evaluación del trabajo implementados de manera remota y automatizada por parte de las plataformas digitales como Uber. Estos sistemas de monitoreo y evaluación 
del trabajo son utilizados para el almacenamiento y explotación de datos de los conductores y las rutas a través de elementos como el GPS, la cantidad de horas que se conectan a la aplicación, las rutas que realizan, los viajes que rechazan y la calificación recibida por parte de los usuarios. Este almacenamiento y explotación de datos ha sido señalado como la principal apuesta de Uber para la generación de su ganancia (Boccardo, 2019; Srnicek, 2016), a través de acciones como la segmentación del mercado, el mejoramiento de los mecanismos de control, conocer mejor las preferencias de los usuarios y desarrollar automóviles auto pilotados, entre otras.

Lo que no se debe olvidar, es que el control no es realizado por un algoritmo, sino que este es solo el medio por el cual el control es llevado a cabo por parte de la empresa, de lo contrario solo se volvería un imperativo tecnológico y se perdería el carácter de clase inherente a la relación laboral (Castillo \& Ratto, 2018). Es más, se ha estudiado que las empresas que operan a través de plataformas digitales se aprovechan de la opacidad de los algoritmos y de las asimetrías de información con respecto a los trabajadores para poder sacar mayor provecho y aumentar las ganancias (Rosenblat \& Stark, 2016).

Si bien algunas investigaciones han señalado que el control algorítmico es capaz de realizar todas las formas de control estudiadas anteriormente a través de la implementación de las denominadas "6 R's" (restringir, recomendar, registrar, rankear, reemplazar y recompensar) (Kellogg et al., 2020), en esta investigación se opta por seguir utilizando las nociones clásicas de control y comparar su aplicación con la del control algorítmico.

De manera que el marco conceptual con el cual se observaron los resultados obtenidos en esta investigación responde principalmente a los desarrollos de la teoría del proceso de trabajo aquí descritos, incorporando los elementos actuales de la discusión propios del contexto específico que se está estudiando e intentando propiciar la comparación entre formas novedosas y persistentes de control presentes en el proceso de trabajo de los conductores de Uber.

\section{Metodología}

Para el desarrollo de esta investigación se entrevistaron a 12 conductores de Uber residentes en la Región Metropolitana de Chile, durante septiembre de 2019 y febrero de 2020. Los criterios muestrales fueron sexo, poseer o no otro trabajo además de Uber y pertenecer o no a organizaciones de conductores de plataformas. Este último criterio fue incorporado debido a que uno de los objetivos de la investigación consistía en comparar las resistencias al control entre trabajadores organizados colectivamente y trabajadores no organizados, cuestión que no se incluye en este artículo.

Los entrevistados fueron contactados mediante grupos en redes sociales y conocidos en común. Luego de realizar las entrevistas a los primeros conductores, 
se les solicitó poder otorgar el contacto de otro conductor, según las características muestrales que se requerían, hasta completar la muestra de 12 entrevistados.

Producto de la alta proporción de hombres dentro de la totalidad de conductores de Uber, se decidió que estos posean una mayor representación en la muestra. De manera que se incluyeron dos conductores hombres según cada criterio, mientras que tan solo una mujer por cada uno. La muestra final quedó estructurada de la siguiente manera:

Tabla 1. Muestreo de la investigación.

\begin{tabular}{|c|c|c|c|}
\hline Número & Sexo & $\begin{array}{c}\text { Pertenencia a aso- } \\
\text { ciación de conductores }\end{array}$ & Trabajo único \\
\hline E1 & Hombre & Pertenece & No posee otro trabajo \\
\hline E2 & Hombre & Pertenece & No posee otro trabajo \\
\hline E3 & Hombre & No pertenece & No posee otro trabajo \\
\hline E4 & Hombre & No pertenece & No posee otro trabajo \\
\hline E5 & Hombre & No pertenece & No posee otro trabajo \\
\hline E6 & Hombre & No pertenece & Posee otro trabajo \\
\hline E7 & Hombre & No pertenece & Posee otro trabajo \\
\hline E8 & Hombre & No pertenece & Posee otro trabajo \\
\hline E9 & Mujer & Pertenece & No posee otro trabajo \\
\hline E10 & Mujer & Pertenece & No posee otro trabajo \\
\hline E11 & Mujer & No pertenece & No posee otro trabajo \\
\hline E12 & Mujer & No pertenece & Posee otro trabajo \\
\hline
\end{tabular}

Fuente: Elaboración propia.

La técnica de producción de información utilizada fue la entrevista semi estructurada. Esto fue adecuado puesto que posee la estructuración necesaria para que el habla de los conductores de Uber pudiera emerger y dar cuenta de su percepción con respecto al control y la relación con la plataforma en general, así como también otros aspectos de su relación laboral que consideraron relevantes.

La información fue analizada utilizando las técnicas de análisis de contenido cualitativo y análisis del discurso. Gracias a esto pudo entenderse tanto los mecanismos de control a los cuales son sometidos los conductores y las prácticas de resistencia a este, como también el sentido otorgado a estas prácticas, considerando que en general los conductores señalaron poseer altos grados de autonomía y flexibilidad sobre la realización de su trabajo. La codificación y análisis de la información se realizó utilizando el software de análisis cualitativo Atlas Ti 7. 


\section{Resultados}

En este apartado se describen y analizan las formas de control de la plataforma Uber sobre sus conductores asociados, teniendo como marco analítico la tipología de control construida a partir de las elaboraciones de los teóricos del proceso de trabajo. En líneas generales, se aprecia una combinación de diversas formas de control, en las cuales se pueden observar elementos novedosos introducidos por la organización del trabajo llevada a cabo por las plataformas, como también elementos de larga data y formas de control persistentes a lo largo de la historia. Es claro el predominio de formas de control basadas en otorgar autonomía sobre los detalles de la realización del trabajo, es decir, horarios, lugares, jornadas, etc., al mismo tiempo que se implementan mecanismos de control remotos para direccionar la forma de realizar el trabajo de los conductores, como también calificar su desempeño. Como se describirá, la estrategia de control de Uber pareciera tener una doble finalidad. Por una parte, que los conductores trabajen la mayor cantidad de tiempo posible, lo que permite aumentar la ganancia de la empresa a través de la monopolización del mercado de transporte privado de pasajeros y el almacenamiento de datos de los conductores y usuarios. Mientras que al mismo tiempo se disminuyen las hostilidades y se genera conformidad con los objetivos de la empresa a través de la flexibilidad de los horarios y jornadas.

\section{Flexibilidad y autonomía en la relación laboral}

Una de las principales manifestaciones del control que ejerce Uber sobre los conductores radica en la flexibilidad y autonomía que poseen estos para definir elementos de su jornada de trabajo, lo cual puede ser entendido como una aplicación de la estrategia de autonomía responsable. Esta forma de control se fundamenta en otorgar grados relativos de autonomía a los trabajadores en la realización de su labor, lo cual tiene por objetivo generar una identificación de estos con los objetivos de la empresa, disminuyendo las hostilidades y previniendo formas de resistencia asociadas a la organización y manifestaciones colectivas (Friedman, 1977). Si bien originalmente esta estrategia fue diseñada para trabajadores calificados a los cuales se les entrega diversos beneficios en función de obtener un compromiso con los objetivos de la empresa, resulta adecuado aplicarlo en este contexto teniendo los resguardos necesarios, considerando que la valoración de la autonomía otorgada en la realización del trabajo a los conductores, representada en la flexibilidad para elegir la cantidad, horarios y lugares de trabajo, es la mayor fuente de consentimiento de los conductores hacia el funcionamiento de la plataforma, haciendo propio el discurso de esta.

Ya se ha descrito la importancia que Uber le otorga en su discurso al hecho de que los conductores no posean ningún vínculo laboral con la plataforma, lo que en su lógica posibilita los altos grados de autonomía y flexibilidad que gozan los conduc- 
tores, cuestión que señalan no sería posible en caso de existir una vinculación más formal tal como un contrato de trabajo. Encuestas y estudios anteriores (CNP, 2018; Fielbaum \& Tirachini, 2019) han señalado que la flexibilidad en el trabajo es uno de los aspectos mejor valorados por los conductores de plataformas, cuestión que se ve comprobada en lo señalado por los conductores al respecto:

"Una de las ventajas de este trabajo es que uno se auto exige, se autocontrola y se genera una rutina de trabajo de acuerdo a la experiencia que haya tenido, monetaria, porque en este caso la demanda te la va dando el pasajero" (E1).

Como se observa, no existe una percepción de estar siendo controlado por la plataforma ni que ésta les exija nada en cuanto a su forma de trabajar. Esta percepción de autocontrol posibilitada por la flexibilidad otorgada por Uber es algo generalizado entre los conductores, quienes recalcan la libertad que poseen para elegir su forma de trabajo:

"No, lo manejo yo. Ese tiempo lo manejo yo. Yo decido cuando salgo y cuando no salgo nomás. No...es absolutamente libre" (E3).

Las respuestas anteriores dan cuenta de la eficacia de la estrategia de autonomía responsable, ya que se logra observar que el discurso de la libertad y autonomía en el trabajo es tomado como propio por gran parte de los conductores. Como se señaló, esta estrategia tiene por objetivo final el controlar la emergencia de posibles descontentos y organización de los trabajadores en contra de los intereses de la empresa (Friedman, 1977). En tal sentido, uno de los principales objetivos que poseen las plataformas es evitar la regulación laboral, lo que le significaría incorporar costos asociados a la fuerza de trabajo. Esta defensa de la inexistencia del vínculo laboral y de negarse a ser regulados también penetra el discurso y la percepción de algunos conductores, quienes defienden la condición en la que se encuentran:

"Si nos vamos a la regla de quién soy dependiente, yo no soy dependiente de Uber, yo hago uso de una aplicación, un servicio o un producto que ellos venden y yo soy un usuario de ese producto" (E1).

Esto es reafirmado por otros conductores, quienes defienden incluso más fuertemente este aspecto frente a las demandas por regulación:

"Al final Uber lo bueno que te da es esa libertad de ser tu propio jefe, obviamente llevándose algo a cambio, pero es parte de cuando uno trabaja en algo independiente, yo encuentro como entre comillas 'abusador', pero entre comillas, querer exigirle algo a alguien que te da el beneficio de poder trabajar libremente" (E4). 
De forma que la estrategia de autonomía responsable implementada por Uber orientada a disminuir las hostilidades y a producir consentimiento en torno a sus objetivos y forma de organización es altamente efectiva. Debido a la gran defensa que algunos conductores realizaban de su condición laboral, en sus entrevistas se incorporaron preguntas que indagaban directamente en su percepción sobre los aspectos negativos que esta condición acarreaba, tal como las largas jornadas de trabajo, el no recibir un sueldo fijo, no poseer cotizaciones previsionales, entre otros. Al respecto, si bien existían variaciones según cada conductor, logra distinguirse una defensa persistente:

"O sea, te revientas 5 días trabajando, si, es verdad, porque trabajar de 8 a 9 es súper pesado, pero tiene sus beneficios, o sea, no trabajas gratis" (E4).

Además, en muchos casos esta defensa se fundamentaba fuertemente en la comparación de Uber con otros trabajos y la realidad del mercado laboral chileno:

“Te dan un montón de facilidades, es súper fácil entrar a Uber, te sacas una foto, le sacas a los papeles de antecedentes, sacas una foto carnet, tu licencia de conducir, el permiso de circulación, vas a que te activen y se acabó, o sea, estás trabajando, yo creo que en ninguna parte vas a encontrar trabajo tan rápido más encima a buenos precios" (E4).

Cuestión que también opera de cierta forma como resignación frente a una realidad que les ha tocado vivir tanto dentro como fuera de Uber:

"Pero eso que tú mencionas de estar fuera de la ley, para la clase obrera no significa nada, no significa nada porque estamos acostumbrados a trabajar harto" (E1).

Ciertamente un análisis de la realidad laboral del país que permite la fácil inserción de plataformas digitales como Uber es interesante de realizar, sin embargo, excede las posibilidades y objetivos planteados en esta investigación. De manera que basta decir que una de las fuentes de consentimiento identificada refiere a la realidad laboral chilena, observando a Uber como una salida a esta realidad.

Algo que llama profundamente la atención es que son los mismos conductores que resaltan la flexibilidad otorgada por Uber, quienes en otras respuestas señalan que en orden de generar los ingresos necesarios para subsistir, deben trabajar los siete días de la semana en jornadas cercanas a las doce horas diarias, lo que invita a cuestionar la materialidad de la flexibilidad ofrecida, la cual parece operar flexibilizando el límite superior de la jornada de trabajo, pero no de forma contraria. Claramente no es el propósito de esta investigación cuestionar la coherencia interna de los discursos de los conductores, pero es interesante analizar este elemento que pareciera paradójico considerando la eficacia de las formas de control asociadas a la autonomía responsa- 
ble y que ha sido reportado por investigaciones similares en otros países (Madariaga, Buenadicha, Molina \& Ernst, 2019).

En particular, es útil volver a introducir las precauciones señaladas por Wood (2019), quien advierte la facilidad con la que se pueden pasar por alto las formas de control general cuando se otorga autonomía sobre los detalles de la realización del trabajo. En este caso, son los mismos conductores quienes conociendo la realidad de sus condiciones de trabajo e incluso desplegando formas de resistencia frente al control que son sometidos, hacen propio el discurso de la flexibilidad y autonomía pregonado por la plataforma.

Como ya se describió, el principal objetivo de la estrategia de autonomía responsable es la reducción de hostilidades y la inhibición de formas de descontento y organización colectiva, por lo que es necesario introducir al análisis las acciones de control desplegadas por Uber cuando estos elementos comienzan a hacerse visibles. Esto es identificado por algunos conductores, en particular aquellos que ya pertenecen a dichas organizaciones y que han sido sancionados de alguna forma por ello:

"Cuando los conductores permanecen mucho tiempo trabajando para las aplicaciones, 3, 4, 5 años, donde ya conocen el funcionamiento, los conductores se empiezan a asociar, se empiezan a sindicalizar, empiezan a reclamar y eso es lo que Uber siempre trata de evitar, la relación que ellos tienen con el conductor vía aplicación es una relación 1 a 1, ellos no quieren que existan relaciones grupales o colectivas" (E2).

Si bien los conductores señalan que las sanciones no son tan drásticas como una desvinculación de la plataforma, si existe una diferenciación entre aquellos conductores que se han organizado y manifestado su descontento y aquellos que no. Esta diferenciación se manifiesta en aspectos como un monitoreo más estricto de los líderes de las organizaciones o en aspectos del funcionamiento de la plataforma, como la oferta de incentivos. $\mathrm{Al}$ respecto, algunos conductores señalan que la plataforma los "marca" de alguna forma para diferenciarlos de los demás:

"A mí no me incentiva, porque yo estoy marcada jajaja" (E9).

La segmentación de los trabajadores entre aquellos que gozan de mayor autonomía y otros que son controlados de forma más estricta y directa es típica de la estrategia de autonomía responsable (Friedman, 1977). Sin embargo, estas formas de control desplegadas por Uber parecieran oscilar entre distintos tipos de control identificados en la tipología construida, ya que por una parte pueden identificarse como parte de la estrategia de autonomía responsable en tanto están orientados a desincentivar la organización colectiva, mientras que por otra parte operan como una aplicación arbitraria de la autoridad, típica de las formas directas de control. 
Lo cierto es que resulta claro que la plataforma cuenta como uno de sus objetivos primordiales el desincentivar la organización colectiva de los conductores que pudiera atentar contra su modelo de organización del trabajo. En tal sentido, una de las principales amenazas a la organización del trabajo realizada por Uber ha sido la regulación estatal implementada en diversos lugares del mundo y que incluso ha llevado a su retirada en algunos países (Williams, 2018). Es por esto que Uber ha intentado eludir la regulación en el país, constituyéndose como un grupo de interés en la discusión sobre la ley de regulación a las plataformas digitales que actualmente se encuentra en tramitación. Al respecto, algunos conductores señalan que la plataforma incluso les ha pedido hacerse parte de manifestaciones:

"Una vez nos mandaron un correo, cuando recién salió, cuando se aprobó la discusión en el Senado, nos mandaron un correo pidiendo que poco menos que protestáramos por la ley, porque si no iban a quedar 80 mil conductores sin trabajo, iban a subir, se iban a triplicar los valores de las tarifas, así un correo del terror, del terror" (E11).

Esto reafirma el hecho de que Uber necesita de su modelo de organización del trabajo para poder generar ganancias, por lo cual necesita controlar las manifestaciones de descontento y cualquier intento de manifestación o regulación.

\section{Normas de comportamiento, evaluación y sanciones arbitrarias}

Otro de los elementos principales del control desplegado por la plataforma Uber sobre el trabajo de sus conductores asociados dice relación con ciertas normas de comportamiento que son aceptadas por los conductores a la hora de establecer su vínculo con la plataforma. Estas normas pueden ser utilizadas para diversos propósitos, desde recomendaciones a los conductores, hasta bloqueos permanentes. En relación con estas normas de comportamiento, la plataforma despliega mecanismos de monitoreo para verificar su cumplimiento, realizando una constante evaluación del desempeño de los conductores y aplicando sanciones de ser necesario, muchas de las cuales son consideradas arbitrarias por parte de los entrevistados.

Estas formas de control pueden enmarcarse dentro de lo que se entiende como control burocrático, puesto que responden a reglas y mandatos establecidos de antemano por la empresa para el desarrollo del trabajo, a las cuales esta se remite en caso de algún conflicto o mala evaluación de la labor realizada. Sin embargo, se observan también ciertas aplicaciones de sanciones de manera arbitraria, las cuales no tienen en cuenta necesariamente el sistema de reglas definido por la plataforma y que por lo tanto pueden ser entendidas como manifestaciones de control simple.

Partiendo por los elementos clasificados dentro del control burocrático, se destaca la existencia de un manual de convivencia que la plataforma pone a disposición de los 
conductores cuando estos firman su vinculación. Este manual regula aspectos referidos principalmente a la relación de los conductores con los pasajeros:

"La conversación, el que tú tengas que ser amable, el que tú tengas que tener tu auto limpio" (E9).

Sin embargo, la gran mayoría de los conductores entrevistados señalaron no conocer la existencia de dicho manual, al mismo tiempo que quienes conocían de su existencia manifestaron nunca haberlo leído, lo que invita a cuestionar su eficacia como forma de control.

A pesar de que el manual parece ser una herramienta poco eficaz de control, lo cierto es que las normas sugeridas en él actúan de forma bastante estricta en la operación de la plataforma, incluso generando que esta se comunique de forma directa con conductores que han pasado a llevar alguna de ellas. Esta situación es manifestada como algo común por parte de los conductores:

"Que de repente dice toma estas consideraciones, mantén el auto limpio, escucha música a un volumen moderado..." (E7).

Así como también:

“De repente te pueden llegar ponte tú, sugerencias de que tu actitud en ciertas cosas, así como súper sutil queriendo decir que no sé po, que la música o que la limpieza o que tu actitud" (E10).

De manera que a pesar de que el manual no es tenido en consideración por la mayoría de los conductores, su contenido sí es importante para la plataforma a la hora de controlar su comportamiento. La no consideración de los aspectos normados por la plataforma puede acarrear no tan solo un mensaje de recomendación, sino que incluso bloqueos permanentes por parte de la plataforma. En relación a eso, un conductor que fue bloqueado por parte de Uber señala:

"Fue una vez que supuestamente yo me robé un teléfono, lo cual no fue, ¿cachai? (sic) Me bloquearon la cuenta, y yo dije no po hueón (sic), si tenía que yo ir y dar mi historia ¿cachai? (sic) Y me dijeron no, a usted cuando le pasen este tipo de situaciones, usted tiene que mandar un correo automáticamente..." (E8).

Mientras que otros conductores cuentan experiencias de las que se han enterado:

"Por auto sucio, auto sucio han cancelado hartas cuentas, choferes que son cascarrabias, las cierran por eso, porque no les sirve po, a ellos les sirve que el pasajero se sienta ameno con el conductor" (E6). 
La forma en la que la plataforma logra monitorear que los conductores cumplan con las normas señaladas en el manual de convivencia es a través de su sistema de calificación. Uber realiza desvinculaciones automáticas a conductores con una calificación menor a 4.7 (CNP, 2018), así como también en caso de que se repitan comentarios por situaciones como el volumen de la música, la limpieza del vehículo, la cordialidad con el pasajero, entre otras.

A pesar de que el manual supuestamente solo contiene sugerencias para los conductores y que su lectura no es obligatoria, las normas que contiene son importantes para la plataforma, llegando incluso a cancelar cuentas producto del incumplimiento de estas normas. La importancia del control de la relación entre el conductor y el pasajero para la plataforma se relaciona con la necesidad de monopolizar el sector por parte de las plataformas digitales, las cuales son dependientes de los efectos en red para generar su ganancia (Srnicek, 2016).

Otro aspecto que ilustra la eficacia de esta forma de control sobre los conductores es la auto regulación del comportamiento en conformidad con estas normas, aun cuando no lo identifican de esta manera:

"Igual trato de sí cuando salgo, trato de lavar el auto, de aspirarlo, no sé po, si me paro a fumar, me bajo del auto a fumar y después me vuelvo a subir, porque igual queda hediondo el auto a cigarro y pueden reclamar po" (E7).

O también:

"Hay dos tipos de pasajeros, los que no hablan con los Uber y los que hablan po, y uno tiene que respetar eso obviamente, por ejemplo, yo soy bueno para hablar, pero yo estoy ahí en mi trabajo y si el pasajero me habla yo lo hago, todo en un margen de respeto. Y yo creo que eso también ayuda a que te evalúen bien, tener el auto limpio igual, ser cordial, todo eso ayuda" (E5).

Una cuestión típica del control burocrático es que las relaciones de poder y autoridad se difuminan y se vuelven difíciles de observar. En particular en el caso de Uber, esto opera como un sentimiento de impotencia por parte de los conductores, quienes no saben a quién dirigirse o cuál es el procedimiento a llevar a cabo luego de una sanción o en caso de algún problema con la plataforma:

“El grave problema es que no tenemos (...) donde hacer un reclamo para que sea revisado o por lo menos para que se le entreguen los antecedentes, eso es porque la aplicación lo tiene como una norma definida de esa manera" (E2).

En este tipo de casos la plataforma se remite a sus términos de servicio para realizar sus sanciones. Esto, sumado a que no existen supervisores ni instancias físicas en las cuales puedan realizarse reclamos o pedir explicaciones, resulta en que los 
conductores no sepan a quien recurrir y desistan de sus intentos de apelación frente a la plataforma.

Sumado a lo anterior, los conductores entrevistados señalan que muchas veces las sanciones aplicadas por Uber son arbitrarias, pasando a llevar las mismas reglas determinadas por la empresa en los términos de servicio y el manual de comportamiento. Si bien en Uber predomina un sistema de evaluación y recompensas institucionalizado, se pueden observar algunas prácticas de control arbitrarias y dirigidas en contra de cierto tipo de conductores. Así lo señalan algunos conductores pertenecientes a organizaciones colectivas:

"A mí no me incentiva, porque yo estoy marcada jajaja... no me dan bonos, yo estoy segura, ni a mí ni a (...) que somos los que fuimos al congreso la vez anterior" (E9).

Así también lo han notado otros conductores:

"Ayer comprobé que las promociones no son para todos (...) me fui conversando con el cabro y le mostré mi plataforma, todas las promociones que tenía, y él me dijo a mí nunca me ha llegado nada, y lleva el mismo tiempo que yo, no tengo idea en qué irá" (E11).

Pareciera que son algunos conductores marcados negativamente quienes no son parte de estos ofrecimientos por parte de la plataforma, sobre todo aquellos conductores que han participado de manifestaciones en contra de la plataforma o se encuentran organizados colectivamente. Lo anterior demuestra arbitrariedad en el sistema de recompensas por el trabajo, aun cuando este supuestamente opera de forma automática a través del algoritmo utilizado por Uber.

Esta arbitrariedad no opera solo en las recompensas ofrecidas a los conductores, sino que también en cuestiones esenciales del trabajo diario, como la información que se tiene del pasajero a la hora de aceptar o rechazar un viaje. Una conductora señala que la plataforma no le permite saber el modo de pago del pasajero:

"A mí no me muestra, yo cuando voy llegando a buscar al pasajero me muestra recién, cuando el pasajero está arriba, ahí me doy cuenta que el viaje es en efectivo. Entonces, acá hay conductores de primera y segunda categoría” (E9).

Uno de los aspectos que Uber utiliza a su favor para llevar a cabo estas formas directas de control es la opacidad con la que operan sus algoritmos. Debido a que la plataforma no informa el modo de operación de estos, los conductores no conocen con certeza cómo funcionan en aspectos como la asignación de pasajeros, ofrecimiento de recompensas, sanciones o cálculo del costo de un viaje, lo que es aprovechado para disimular la arbitrariedad con que Uber opera en varios de estos aspectos. Esto 
constituye una particularidad de la aplicación del control simple en este contexto, el cual debe ser disimulado, distinto a la simple arbitrariedad en la autoridad típica de esta forma de control.

Como se ha señalado en investigaciones anteriores, las asimetrías de información son fundamentales tanto para que la plataforma pueda implementar formas de control, como también para que los conductores puedan desplegar sus formas de resistencia a dicho control (Rosenblat \& Stark, 2016). A pesar de que esta investigación no profundiza en este elemento en particular, es importante señalar que, en lo referido a las formas directas de control, la plataforma se refugia en la opacidad de los algoritmos para operar arbitrariamente en cuestiones como la dirección de las zonas de trabajo, la recompensa por este y las sanciones y castigos asociados.

Una última forma de control simple implementada por Uber dice relación con la unilateralidad en la toma de decisiones. Si bien los conductores antes de comenzar a conducir con la plataforma deben aceptar ciertas condiciones señaladas en los términos de servicio, estos también señalan que la plataforma actúa unilateralmente y realiza cambios sin avisarles. Una de las cosas que más afecta y molesta a los conductores al respecto es el cambio en el porcentaje de la tarifa que se queda la plataforma:

"Mucha gente lo piensa, o sea, estás como atrapado en una situación donde claro, donde ellos de repente te cobran una comisión $\mathrm{x}$, después te cobran otra, después te cobran otra, pero no hay dónde reclamar" (E10).

La unilateralidad con la que opera Uber está respaldada por su forma de organización del trabajo, ya que legalmente no cuenta con trabajadores a su servicio y puede realizar estos cambios sin necesidad de consultar a los conductores, quienes sienten que no existe un espacio en el cual puedan canalizar sus demandas y reclamos. Este aspecto ha sido resaltado por organismos como la Organización Internacional del trabajo (2019) y es uno de los que mayor molestia genera entre los conductores, siendo una de las principales razones por la que se han organizado colectivamente en Chile y en el mundo.

\section{Recompensas, incentivos y rastreo remoto}

Otro aspecto del control que emerge de forma clara en el discurso de los conductores refiere a que elementos como la frecuencia con la que aceptan viajes o las horas que le dedican al trabajo no son planificados por ellos de forma premeditada, sino que responde directamente a las vinculaciones con pasajeros que la plataforma les muestra durante su jornada, principalmente a través de incentivos y promociones. De esta forma lo señala una conductora:

"Yo me subía al auto, me conectaba y como que al tiro me empezaban a salir viajes, entonces como que de alguna u otra manera los viajes se iban dando 
solos. Entonces no había mucha planificación, yo como que trataba de conectarme y empezar a ir a buscar hacia lo que te digo yo, pero la verdad es que el viaje como que se daba solo, los trayectos se daban solos" (E12).

También influenciando en cuestiones como los descansos entre viajes o la duración de la jornada:

"No paró de sonar y toda la noche con tarifa dinámica. Así tun, tun, tun (sic) todo el rato, o sea, si paraba para pasar acá al baño, qué sé yo, me tenía que desconectar y pasar ¿me entiendes? O seguía sonando" (E10).

Es posible entender estos elementos como aplicaciones del control técnico, el cual se caracteriza por la vinculación directa del ritmo e intensidad del trabajo con la tecnología utilizada para la producción (Edwards, 1978). A diferencia del control simple en donde la intensidad del trabajo es controlada de forma directa por supervisores, el control técnico se caracteriza porque el ritmo, intensidad y cantidad de trabajo son controlados por la tecnología implementada por la empresa. En tal sentido, las empresas intentan implementar tecnologías que faciliten la efectiva conversión de la fuerza de trabajo en trabajo real. Sin embargo, nuevamente es problemático encasillar este tipo de acciones en tan solo una forma de control, puesto que también podría entenderse perfectamente como aplicaciones del control algorítmico, en tanto opera de manera automatizada generando incentivos y recompensas para influir en la jornada de trabajo de los conductores.

Con todo, lo cierto es que los conductores dan cuenta de que su ritmo e intensidad de trabajo depende directamente de las indicaciones señaladas por la plataforma en forma de incentivos y recomendaciones, tales como las denominadas tarifas dinámicas o las recompensas por realizar una determinada cantidad de viajes en un período específico de tiempo. Esta situación se acentúa sobre todo cuando se necesita generar mayores ingresos que lo habitual, puesto que los conductores se muestran más dispuestos a aumentar el ritmo y cantidad de trabajo:

"Yo no alcanzo a terminar una carrera y llega enseguida otra y otra y otra, y así estoy, puedo estar todo el día, si no me desconecto no paro. Yo lo máximo que he trabajado han sido 13 horas de corrido y gané 100 lucas" (E11).

También en relación a la necesidad de generar mayores ingresos, la empresa otorga promociones a los conductores que funcionan como incentivos por cantidad de viajes realizados en una cierta cantidad de tiempo o en un determinado horario. Estas promociones también operan como una forma de control técnico sobre la intensidad con la que los conductores deben realizar su trabajo, puesto que la cantidad de viajes que se necesitan realizar en una cantidad específica de tiempo obliga a los conductores a aceptar un viaje tras otro. Esto señala un conductor al ser consultado sobre si las promociones ofrecidas son posibles de realizar: 
"Es complicadísimo, lo hacíamos justo, de repente yo lo hacía justo, pero te dedicabas ¿cachai? (sic) o sea, ni siquiera tenías tiempo para tomarte una bebida, tenías que hacer carrera tras carrera, carrera tras carrera" (E6).

Estas promociones tienen como condición que los conductores no rechacen viajes dentro del horario establecido para poder ganar el bono, lo que también afecta directamente la intensidad del trabajo y la autonomía de los conductores sobre su forma de trabajar:

"Me exigen por ejemplo el tema de los viajes consecutivos para darme las promociones. Me dicen 'ya, tienes que cumplir primero sin desconectarte ni rechazar ningún otro viaje', porque ahí tampoco cumple la promoción, o sea, tienen que ser los 3 viajes siempre conectado y sin rechazar nada, si yo rechazo algo me dicen 'entonces, no"' (E5).

Como se observa, la plataforma logra controlar el ritmo con el que los conductores toman viajes, influenciando sus planificaciones y manejo del tiempo de trabajo. De esta forma la plataforma logra maximizar el tiempo de trabajo de sus conductores asociados, limitando los tiempos muertos y las pausas entre viajes.

Podría argumentarse que los conductores son libres de desconectarse de la aplicación en el momento que ellos quieran o de rechazar los viajes hacia lugares donde no quieren ir. Sin embargo, la plataforma también implementa mecanismos que permiten controlar estos aspectos, puesto que genera sanciones a partir de la tasa de cancelación y de rechazo de viajes de los conductores:

"Tú no puedes cancelar la carrera, si cancelas la carrera te bloquean la cuenta. Si cancelas una carrera, te la bloquean por 1 minuto, cancelas otra carrera te la bloquean por 3 horas (...) cancelas otra carrera y te la bloquean por 24 horas y cancelas otra y te la bloquean para siempre, ¿cachai? (sic) si es un tema también" (E6).

De manera que es necesario tener en cuenta estas sanciones a la hora de evaluar la autonomía de los conductores sobre su forma de trabajar. Además, es imposible obviar las condiciones que se presentan como imperativos a la hora de decidir estos aspectos del trabajo. Son los mismos conductores quienes tienen esto en consideración:

"Al principio, como era muy vicioso el trabajo, hubieron (sic) hartos colegas que se accidentaron por la falta de sueño y lo mismo, que es un sistema que te enrolla, o sea, todas estas aplicaciones (...) es un sistema que te obliga y te va manipulando a que te cautive, de no salirte, es como entrar a ludopatía, o sea, te metes y empieza a sonar, a sonar, a sonar y hasta el sonido creo yo que está estudiado, hasta el sonido te genera sonido de plata” (E1). 
Muchos conductores logran identificar alguna forma de control o manipulación de la plataforma sobre algún aspecto de su trabajo. Sin embargo, es difícil lograr escapar a dicho control debido a la necesidad de generar dinero. De esta forma, la plataforma logra generar una aparente coincidencia entre los intereses y objetivos de los conductores y los de la plataforma, argumentando a favor de la necesidad de realizar la mayor cantidad de viajes posibles y logrando controlar aspectos relacionados a la forma de llevar a cabo el trabajo por parte de los conductores, aun cuando el lenguaje de la flexibilidad y la autonomía sean la punta de lanza de Uber.

Otro de los problemas que esta forma de control trae aparejado y que la literatura ha destacado (Graham \& Hjorth, 2017; OIT, 2019) es la extensión de las jornadas de trabajo por sobre los límites legales, generando peligros asociados a la conducción por grandes periodos de tiempo y afectando el tiempo de ocio de los trabajadores. Esto es reafirmado por una conductora que sufrió un accidente mientras conducía con la plataforma:

“...Me quedé dormida, choqué y me quedé dormida (...) yo venía de dejar a dos pasajeros en Chicureo que pidieron calefacción y ahí me morí y eran las 5:43 más o menos, y justo iba subiendo la pirámide, acepté el viaje y pum (sic), no me preguntes qué me pasó, cerré los ojos y me fui contra la pared. No, es que nos pasa, porque estamos trabajando 12 horas po" (E9).

Finalmente, otra de las manifestaciones cruciales del control que experimentan los conductores refiere al rastreo remoto que la plataforma ejerce sobre ellos. Esta posibilidad está dada porque los conductores conectados son visibles en el mapa de la aplicación, por lo que sus recorridos son almacenados como información, siendo posible observar la localización de cada conductor conectado. Más allá de los usos que Uber posea para dicha información, resulta claro que la plataforma solo puede rastrear la ubicación del automóvil sin tener certeza de quién lo está conduciendo, cuestión que ha sido aprovechada por algunos conductores que venden o arriendan sus cuentas, principalmente a extranjeros que no han regularizado su situación migratoria y por lo tanto no pueden crear una. Es a partir de esta situación que los conductores señalan que la plataforma ha desplegado otras formas de rastreo para cerciorarse que quien conduce el automóvil sea efectivamente el titular de la cuenta:

"Si, sacarme una foto antes de manejar, ahora que he dejado de manejar harto tiempo. Onda, cuando partí como las primeras 4 veces conectado, me decía 'por favor tómate una foto antes de iniciar la aplicación"' (E6).

Lo que muchos conductores interpretan como una medida de seguridad implementada por la plataforma: 
"A mí me lo ha pedido muchas veces, una selfie y eso es una política de seguridad para que sea realmente el conductor, el de la foto, el que va manejando" (E9).

Sin cuestionar que esta acción pueda operar efectivamente como una medida de seguridad, resulta claro que es una forma de control sobre los conductores, utilizando el reconocimiento facial para cerciorarse que es el conductor quien se encuentra conduciendo. Lo anterior, también trae problemas relacionados a la información personal que la plataforma almacena a través del reconocimiento facial y los usos que se le pueda dar a dicha información, los cuales van más allá del consentimiento que se pueda otorgar al aceptar conducir para la plataforma.

De esta forma, Uber logra rastrear a sus conductores activos, controlando que la aplicación sea utilizada de la forma indicada y almacenando información utilizada para el funcionamiento de sus algoritmos y otros fines orientados a generar ganancia a partir de la explotación de los datos, como la literatura al respecto ha señalado (Boccardo, 2019; Srnicek, 2016).

Para finalizar, se presenta un cuadro resumen de las principales formas de control desplegadas por la plataforma Uber, clasificadas según la tipología conceptual construida:

Tabla 2. Formas de control identificadas.

\begin{tabular}{|l|l|}
\hline Control simple & $\begin{array}{l}\text { Las formas de control simple son más bien resabios, producto de la } \\
\text { progresiva desaparición de este tipo de control en favor de sistemas } \\
\text { de evaluación, sanción y recompensa del trabajo más institucio- } \\
\text { nalizados. En particular se encuentran sanciones arbitrarias contra } \\
\text { conductores que se han involucrado en manifestaciones de descon- } \\
\text { tento y líderes de organizaciones colectivas, además de cambios uni- } \\
\text { laterales en los términos de servicio de la plataforma. Se aprovecha } \\
\text { la opacidad de los algoritmos para desplegar esta forma de control, } \\
\text { al contrario de la arbitrariedad abierta que se ha encontrado en el } \\
\text { pasado en otros contextos. }\end{array}$ \\
\hline Control técnico & $\begin{array}{l}\text { El control técnico opera en relación a la intervención de la tecnología } \\
\text { implementada por la plataforma en el ritmo de trabajo de los con- } \\
\text { ductores. Se presenta fundamentalmente en la fijación del ritmo de } \\
\text { trabajo a partir de la vinculación con pasajeros y en las promociones } \\
\text { ofrecidas por trabajar en ciertos horarios y lugares, influyendo en la } \\
\text { cantidad de tiempo dedicado al trabajo, en los descansos entre viajes } \\
\text { y el estilo de trabajo en general. }\end{array}$ \\
\hline
\end{tabular}




\begin{tabular}{|c|c|}
\hline $\begin{array}{l}\text { Control } \\
\text { burocrático }\end{array}$ & $\begin{array}{l}\text { Las formas de control burocrático son limitadas producto del con- } \\
\text { texto específico del trabajo en Uber, distinto al original en donde esta } \\
\text { forma de control se aplicaba a trabajadores calificados con una carre- } \\
\text { ra estable dentro de una misma empresa. Su principal manifestación } \\
\text { es la aplicación de sanciones a partir de normas establecidas de ante- } \\
\text { mano y posibilitadas por el sistema de evaluación a los conductores, } \\
\text { además de la difuminación de las relaciones de autoridad a partir de } \\
\text { la referencia hacia los términos de servicio y el manual de conviven- } \\
\text { cia en caso de reclamos y descontento. }\end{array}$ \\
\hline $\begin{array}{l}\text { Autonomía } \\
\text { responsable }\end{array}$ & $\begin{array}{l}\text { Si bien esta estrategia de control fue pensada para trabajadores cali- } \\
\text { ficados y de rango medio, otorgando beneficios a cambio de compro- } \\
\text { miso con la empresa, opera de forma bastante eficaz en el contexto } \\
\text { del trabajo en Uber. En particular funciona otorgando flexibilidad en } \\
\text { el trabajo a cambio de consentimiento con la forma de organización } \\
\text { de la plataforma, siendo la principal fuente de consentimiento halla- } \\
\text { da. El objetivo principal de esta estrategia de control es la inhibición } \\
\text { de descontentos y manifestaciones, por lo que también actúa de for- } \\
\text { ma rápida y estricta contra este tipo de manifestaciones. }\end{array}$ \\
\hline $\begin{array}{l}\text { Control } \\
\text { algorítmico }\end{array}$ & $\begin{array}{l}\text { El control algorítmico surge particularmente en el contexto de las } \\
\text { plataformas digitales como Uber. Se caracteriza por la posibilidad de } \\
\text { rastreo remoto sobre los conductores y las decisiones aplicadas de } \\
\text { manera automática. A partir de la aplicación de este tipo de control } \\
\text { los conductores son coaccionados a incrementar el tiempo de trabajo } \\
\text { y su comportamiento es monitoreado gracias a herramientas como } \\
\text { el GPS, el reconocimiento facial y las calificaciones de los usuarios, lo } \\
\text { que permite la generación de datos utilizados para mejorar el control } \\
\text { y conocer mejor las preferencias de los usuarios. }\end{array}$ \\
\hline
\end{tabular}

Fuente: elaboración propia.

\section{Conclusión}

De manera que se observa la efectiva existencia de control por parte de Uber sobre sus conductores. Si bien dicha existencia opera como un supuesto de la investigación a partir de los antecedentes recopilados, no está demás insistir en esto, teniendo en consideración aquellas posiciones que acentúan los aspectos asociados a la autonomía, la flexibilidad, la economía colaborativa y lo provechoso que serían estas plataformas tanto para la economía como para los trabajadores. Si bien en el ámbito académico estas posiciones parecen no ser mayoritarias, en el debate público poseen un peso importante, tal como lo demuestran las derivas que ha tomado el debate legislativo que pretende regular a las plataformas en Chile. 
Frente a aquellas posiciones, tanto positivas como negativas, que observan a las plataformas como Uber como un fenómeno completamente novedoso, debe señalarse que en cuanto a control de la fuerza de trabajo se refiere, la plataforma Uber se muestra más bien como una continuación de la forma de organizar el trabajo dentro del capitalismo, caracterizada por la contradicción de intereses entre el empleador y los trabajadores, haciendo inevitable el control de la fuerza de trabajo por parte de la gerencia en función de maximizar la generación de ganancia (Braverman, 1983).

Algunas posiciones críticas respecto al funcionamiento de las plataformas digitales han planteado que lo novedoso no se encuentra en la inexistencia de control, sino más bien en las formas que este control adquiere, acentuando aspectos como la vigilancia tecnológica que se hace total e imposible de eludir, propias de la corriente denominada critical management studies (Thompson, 2009). Como se intenta demostrar en la presente investigación, es necesario también matizar dichas posiciones, producto que el control ejercido por Uber incorpora formas de control antiguas y persistentes a lo largo de la historia, como lo demuestra la incorporación de control directo que, aunque no tan extendido, es desplegado por la plataforma. Asimismo, se observan con mayor o menor intensidad formas de control similares a las identificadas por las investigaciones centradas en el proceso de trabajo a lo largo de los años, asociadas a estrategias incorporadas al menos desde la década de los 50'.

Ahora bien, es necesario señalar que no todo es persistencia en las formas de controlar la fuerza de trabajo poseídas por Uber. Tal como se describió en el capítulo, la plataforma efectivamente incorpora elementos novedosos, asociados tanto a la forma de organización del trabajo como a la introducción de nuevas tecnologías que posibilitan el rastreo remoto y la operación automática de la plataforma en aspectos como la evaluación, recompensas y sanciones.

Si bien entre los mecanismos de control encontrados se encuentran las "6 R's" identificadas en otras investigaciones (Kellogg et al., 2020), lo cierto es que muchos de esos mecanismos no son novedosos del trabajo en plataformas, sino que son elementos de larga data normalmente identificados con otras formas de control. A pesar de que es cierto que el mecanismo a través del cual es aplicado el control cambia gracias a la incorporación de nuevas tecnologías con fines de control de la fuerza de trabajo, no es razón suficiente para señalar que corresponde íntegramente a una forma nueva de control.

Lo que pareciera más adecuado concluir dice relación con una combinación entre elementos de control persistentes a lo largo del tiempo y elementos novedosos incorporados en los últimos años. En específico, esta combinación parece operar como una introducción de mayor eficacia a métodos de control antiguos gracias a las tecnologías ya mencionadas, haciendo que tanto las formas nuevas como antiguas de control sean más instantáneas, opacas y abarcadoras. 
Para finalizar, es necesario introducir ciertas limitaciones de este artículo. En primer lugar, es necesario señalar que un análisis completo del proceso de trabajo en Uber debe tener en cuenta elementos que no fueron considerados aquí, como el modelo de negocios de la empresa, la conflictividad de los trabajadores, la legislación laboral del país, entre otros que no se contemplaron en este artículo en virtud de centrarse específicamente en las formas de control desplegadas por la plataforma.

De la misma manera, esta investigación estuvo diseñada exclusivamente para los conductores de la Región Metropolitana de Chile, siendo construida una muestra acotada en función de aquello. Si bien por momentos pareciera que los resultados y las conclusiones son extrapolables a todo el país, es un sesgo que es necesario tener en cuenta a la hora de ponderar los resultados de esta investigación.

Otra limitación refiere a que esta investigación tuvo lugar entre diversos fenómenos presentes en la sociedad chilena que pudieron haber alterado el objeto de estudio y probablemente lo transformen en el corto plazo. El trabajo de producción de información comenzó poco antes de las movilizaciones sociales de octubre de 2019, viéndose interrumpido por estas y siendo finalizado poco antes del inicio de la pandemia del coronavirus. Estos fenómenos sin duda han introducido elementos novedosos que merecen la pena ser estudiados y que no lograron integrarse a cabalidad. Es de esperarse que transformen diversos aspectos de la sociedad chilena, incluyendo las relaciones laborales y el trabajo en plataformas particularmente. De la misma forma, el proyecto de ley que regula el trabajo en plataformas digitales al cual se ha hecho referencia a lo largo del texto sigue en tramitación, por lo que su promulgación en el corto plazo también transformará el trabajo en plataformas. Todos estos fenómenos señalan la necesidad de actualizar los resultados aquí obtenidos en un periodo relativamente corto de tiempo.

\section{Referencias}

Boccardo, Giorgio (15 de Mayo de 2019). Industria de datos: Tendencias seculares, nuevas desigualdades y desafíos para la democracia. Palabra pública. Recuperado de https://palabrapublica.uchile.cl/2019/05/15/industria-de-datos-tendenciasseculares-nuevas-desigualdades-y-desafios-para-la-democracia/.

Braverman, Harry (1983). Trabajo y capital monopólico. México D.F: Editorial Nuestro tiempo.

Castillo, Alejandro \& Nicolás Ratto (2018). Teorías del proceso de trabajo. Una revisión de su desarrollo y de las nociones de control y resistencia. Documento de trabajo $N^{\circ} 11$. Chile: Fondecyt 1150860 \& CIPSTRA.

Comisión Nacional de Productividad. (2018). Tecnologías disruptivas. Regulación de plataformas digitales. Capitulo 3 Plataformas de transporte. Santiago. 
De la Garza, Enrique (2012). Hacia un concepto ampliado de trabajo. El mundo del trabajo en América Latina.

De Stefano, Valerio (2016). La «gig economy» y los cambios en la protección social. Gaceta sindical. Reflexión y debate, 27, 149-171.

Edwards, Richard (1978). The social relations of production at the point of production. Critical sociology 109 (8): 108-125.

Edwards, Richard (1990). Understanding conflict in the labour process: The logic and autonomy of struggle. En D. Knights, \& H. Willmott, Labour process theory (pp. 125-152). Londres: The Macmillan press.

Fielbaum, Andrés, \& Alejandro Tirachini (2019). Características del trabajo de conducción en plataformas ride-hailing (tipo Uber y Cabify) en Chile. Santiago.

Friedman, Andy (1977). Responsible autonomy versus direct control over the labour process. Capital and Class 1(1): 43-57.

Gandini, Alessandro (2019). Labour process theory and the gig economy. Human relations 72, (6): 1039-1056. Doi: https://doi.org/10.1177\%2F0018726718790002.

Graham, Mark, Isis Hjorth \& Vili Ledonvirtha (2017). Digital labour and development: impacts of global digital labour platforms and the gig economy on worker livelihoods. Sagepub journal, 23 (2): 135-162. Doi: https://doi. org/10.1177\%2F1024258916687250.

Kellogg, Katherine, Melissa Valentine \& Angele Christin (2020). Algorithms at work: The new contested terrain of control. Academy of Management Annals, 14(1): 366-410. Doi: https://doi.org/10.5465/annals.2018.0174.

Madariaga, Javier, César Buenadicha, Erika Molina \& Christoph Ernst (2019). Economía de plataformas y empleo ¿Cómo es trabajar para una app en Argentina? Buenos Aires: CIPPEC - BID - OIT.

Martínez, Paula \& Ignacia Armijo (23 de Octubre de 2018). Trabajo precario y plataformas digitales, un debate pendiente. El Mostrador. Recuperado de https://www.elmostrador.cl/noticias/opinion/2018/10/23/trabajo-precario-yplataformas-digitales-un-debate-pendiente/.

Möhlmann, Mareike, \& Lior Zalmanson (2017). Hands on the wheel: Navigating algorithmic management and Uber's drivers autonomy. International Conference on Information Systems . Seoul.

Organización Internacional del Trabajo (OIT). (2019). Las plataformas digitales y el futuro del trabajo. Cómo fomentar el trabajo decente en el mundo digital. Ginebra. 
Ríos, Cristóbal \& Lucas Cifuentes (07 de Abril de 2020). El relato de los trabajadores de delivery que viven la pandemia sin acceso a baños ni elementos de protección. Ciper Chile. Recuperado de https://www.ciperchile.cl/2020/04/07/el-relato-delos-trabajadores-de-delivery-que-viven-la-pandemia-sin-acceso-a-banos-ni-elementos-de-proteccion/.

Rosenblat, Alex, \& Luke Stark (2016). Algorithmic labor and information asymmetries: A case study of Uber's drivers. International journal of communication, 10, 3758-3784.

Srnicek, Nick (2016). Platform capitalism. John Wiley and sons.

Thompson, Paul (2009). Labour process theory and critical management studies. En The Oxford handbook of critical management studies (pp. 100-122). Oxford: Oxford university press. Doi: https://doi.org/10.1093/oxfordhb/9780199237715.001.0001.

Todolí, Adrián (2015). El impacto de la "Uber economy" en las relaciones laborales: los efectos de las plataformas virtuales en el contrato de trabajo. IUSLAbor, 3, 1-25.

Vaclavik, Marcia \& Liana Pithan (2018). The agency search: the meaning of work for app drivers. Human and social management, 19 (5): 1-27.

Williams, Guido (2018). Uber y conductores. Legislación comparada sobre relación laboral. Biblioteca del Congreso Nacional.

Wood, Alex (2019). The Taylor Review: understanding the gig economy, dependency and the complexities of control. New technology, work and employement, 1-9. Doi: https://doi.org/10.1111/ntwe.12131.

\section{Sobre el autor}

Osvaldo Vallejos Rivero es Sociólogo de la Universidad de Chile, Estudiante de Magíster en Gestión y Políticas Públicas, Universidad de Chile. Correo Electrónico: ovallejos16@gmail.com. (iD) https://orcid.org/0000-0001-7171-6549 


\title{
CUHSO
}

Fundada en 1984, la revista CUHSO es una de las publicaciones periódicas más antiguas en ciencias sociales y humanidades del sur de Chile. Con una periodicidad semestral, recibe todo el año trabajos inéditos de las distintas disciplinas de las ciencias sociales y las humanidades especializadas en el estudio y comprensión de la diversidad sociocultural, especialmente de las sociedades latinoamericanas y sus tensiones producto de la herencia colonial, la modernidad y la globalización. En este sentido, la revista valora tanto el rigor como la pluralidad teórica, epistemológica y metodológica de los trabajos.

\author{
EDITOR \\ Matthias Gloël \\ COORDINADORA EDITORIAL \\ Claudia Campos Letelier \\ CORRECTOR DE ESTILO Y DISEÑADOR \\ Ediciones Silsag \\ TRADUCTOR, CORRECTOR LENGUA INGLESA \\ Aurora Sambolin Santiago \\ SITIO WEB \\ cuhso.uct.cl \\ E-MAIL \\ cuhso@uct.cl \\ LICENCIA DE ESTE ARTÍCULO \\ Creative Commons Atribución Compartir Igual 4.0 Internacional
}

